\title{
Article
}

\section{Phenotyping Metastatic Brain Tumors Applying Spectrochemical Analyses: Segregation of Different Cancer Types}

Bury, Danielle Elizabeth, Faust, Guy, Paraskevaidi, Maria, Ashton, Katherine M., Dawson, Timothy P. and Martin, Francis L

Available at http://clok.uclan.ac.uk/22834/

Bury, Danielle Elizabeth, Faust, Guy, Paraskevaidi, Maria, Ashton, Katherine M., Dawson, Timothy P. and Martin, Francis L ORCID: 0000-0001-8562-4944 (2019) Phenotyping Metastatic Brain Tumors Applying Spectrochemical Analyses: Segregation of Different Cancer Types. Analytical Letters, 52 (4). pp. 575-587. ISSN 0003-2719

It is advisable to refer to the publisher's version if you intend to cite from the work. http://dx.doi.org/10.1080/00032719.2018.1479412

For more information about UCLan's research in this area go to

http://www.uclan.ac.uk/researchgroups/ and search for <name of research Group>.

For information about Research generally at UCLan please go to http://www.uclan.ac.uk/research/

All outputs in CLoK are protected by Intellectual Property Rights law, including Copyright law. Copyright, IPR and Moral Rights for the works on this site are retained by the individual authors and/or other copyright owners. Terms and conditions for use of this material are defined in the policies page. 

列

3

\title{
Phenotyping metastatic brain tumours applying spectrochemical analyses: segregation of different cancer types
}

\author{
Danielle Bury ${ }^{1, *}$, Guy Faust ${ }^{2}$, Maria Paraskevaidi ${ }^{1}$, Katherine M. Ashton ${ }^{3}$, Timothy P. \\ Dawson $^{3}$ and Francis L. Martin ${ }^{1, *}$ \\ ${ }^{1}$ School of Pharmacy and Biomedical Sciences, University of Central Lancashire, Preston \\ PRI 2HE, UK \\ ${ }^{2}$ Department of Oncology, University Hospitals of Leicester NHS Trust, Leicester, \\ Leicestershire, $U K$ \\ ${ }^{3}$ Department of Neuropathology, Royal Preston Hospital, Lancashire Teaching Hospitals \\ NHS Trust, Sharoe Green Lane, Preston PR2 9HT, UK
}

Email addresses:

Deb11@doctors.org.uk, Guy.Faust@uhl-tr.nhs.uk, mparaskevaidi@uclan.ac.uk, katherine.ashton@1thtr.nhs.uk, timothy.dawson@lthtr.nhs.uk, flmartin@uclan.ac.uk (1)

"Corresponding Authors: Prof Francis L Martin and Danielle Bury, School of Pharmacy and Biomedical Sciences, University of Central Lancashire, Preston PR1 2HE, UK; Telephone: 01772896482; Email: flmartin@uclan.ac.uk; deb11@ doctors.org.uk

The authors declare no competing interests.

2 All authors have contributed equally. 


\section{Abstract}

Metastatic brain tumours represent a significant proprotion of tumours identified intraoperatively. A rapid diagnostic method, circumventing the need for histopathology studies could prove clinically useful. As many spectroscopic studies have shown ability to differentitate between different tumour types, this technique was evaluated for use within metastatic brain tumours. Spectrochemical approaches [Raman and attenuated total reflection Fourier-transform infrared spectroscopy (ATR-FTIR) spectroscopy] were applied to determine how readily it could identify the primary site from the metastatic tumour. Metastases were from primary adenocarcinomas of lung $(n=7)$ and colorectum ( $n=7)$, and for comparison, metastatic melanoma $(n=7)$. The objective was to determine if Raman or ATR-FTIR spectroscopy could delineate the origin of the primary tumour. The results demonstrate that there are marked similarities between the two adenocarcinoma groups and whilst Raman and ATR-FTIR can distinguish the three groups with limited success, classification accuracy is greatly improved when combining the adenocarcinoma groups. The use of such techniques in the clinical setting is more likely to be found intraoperatively, determining the presence of a tumour and suggesting the tumour class; however, traditional histopathology would still be needed to identify the primary origin of the tumour.

Keywords: Attenuated total reflection Fourier-transform infrared (ATR-FTIR) spectroscopy, classification, linear discrimination analysis (LDA), metastatic brain tumour, neuro-oncology, Raman spectroscopy 
Metastatic brain tumours are usually the end-point in a persons' battle with cancer, yet for some may represent the initial diagnosis. The background prevalence of metastatic brain tumours is difficult to quantify; however, those clinically detectable outnumber intrinsic tumours by roughly 3 to 1 , with the majority of metastases arising from primary lung tumours (Davis et. al. 2012, Huang and Ouyang 2013, Renfrow and Lesser 2013). In contrast, colorectal tumours comprise 4-8\% of metastasis, yet less than $9 \%$ of all cases metastasise to the brain (Sanghvi et. al. 2017).

Up to $15-25 \%$ of brain tumours diagnosed are a metastasis (Bekaert et. al. 2017).

Whilst $80 \%$ of patients have a known primary, for some patients the identification of metastasis may be the initial presentation of the primary tumour (Bekaert et. al. 2017). It is thought that the actual incidence of brain metastases is higher than reported as some may go undiagnosed. For those who undergo metastectomy for diagnosis or symptom relief, the tissue, once removed is sent for histopathological analysis to determine the location of the primary tumour.

Currently, diagnosis generally relies upon a mix of haematoxylin and eosin (H\&E) morphological appearances, special tinctorial stains and immunohistochemical (IHC) tests that enable the pathologist to give either a single or group of organs from which the primary tumour likely arises. Morphologically these tumours can look remarkably similar. However, there remains a group of unclassifiable tumours, which are labelled as 'cancer of unknown primary (CUP)' when histopathology and radiology fails to determine a primary origin. The challenge can then be to determine the most likely primary origin in order to guide cancer specific oncological treatment. In an era where 
cancer treatment is guided more by genetic alterations, such as epidermal growth factor receptor (EGRF) mutations in lung cancer, to enable personalised treatment, the need to determine the primary origin to guide genetic testing has never been more crucial (Kalia 2015).

Over recent years many biomarkers have been suggested for identification of disease and monitoring of disease progression in known cancer patients, such as prostate specific antigen (PSA) in prostate cancer patients. The difficulty, however, is that not all patients with prostate cancer will demonstrate a rise in PSA, nor do all patients with a high PSA have prostate cancer. Whilst it is thought those with prostate cancer and low PSA represents less than $1 \%$ of such patients, as the condition becomes more prevalent this is likely to increase (Lee et. al. 2010). Therefore the ability to have a specific and sensitive marker for tumours is crucial.

In recent years, Raman or attenuated total reflection-Fourier transform infrared (ATR-FTIR) spectroscopy methods have been used to delineate a variety of primary and metastatic tumours with varying success (Theophilou et al. 2015, 2016). Raman and ATR-FTIR spectroscopy are complimentary techniques; Raman spectroscopy detects chemical bonds via scattering of photons due to bond vibrations, whereas ATR-FTIR spectroscopy measures energy absorbance after excitation by an infrared (IR) beam following reflection of the beam via an internal element (often diamond or germanium). Both provide a 'fingerprint' of the elements within the examined tissue, which have been used to differentiate between cancerous and non-cancerous tissue and biofluids within a variety of studies (Owens et. al. 2014). Krafft et al. (2006) were able to determine the primary origin from brain metastases of three tumours using IR spectroscopic imaging with variable success (Krafft et al. 2006). They 
compared normal brain to metastases from lung, colon, breast and renal carcinoma. Results showed tumour primary site could be delineated; however, there was an overlap between breast, lung and colorectal carcinomas. A later study by the same group, again using imaging methods but a broader range of cancers, also demonstrated similar overlap within the adenocarcinomas (Bergner et. al. 2013). Given the relatively similar morphological appearances and IHC staining results overlaps, this is not surprising. Gajjar et. al. (2012) also demonstrated positive results in distinguishing different intrinsic brain tumours from normal brain tissue, demonstrating the ability of Raman and ATR-FTIR spectroscopy to segregate different tumour types (Gajjar et. al. 2012).

Outside of the brain, the use of spectroscopy on both tissue and blood components has shown promise in the detection of many cancers around the body, including skin, oesophagus, ovary and cervix with varying degrees of success (Krafft et. al. 2006, Gajjar et. al. 2012, Lyng et. al. 2007, Lui et. al. 2012, Kendall et. al. 2010, Barr et. al. 2011, Mitchell et. al. 2014). However, relatively few studies focus on the differentiation of primary tumour from metastasis. Therefore, within this study, brain metastasis from lung and colorectal adenocarcinomas have been chosen due to their similar morphological appearances (see Figure 1), and their ability to often have challengingly similar IHC staining patterns. Whilst at first glance these tumours may appear different, it is not possible on morphology alone to determine the definitive primary location of the tumour and immunohistochemistry is regularly performed. This limited variability between the two adenocarcinomas will provide a challenge to determine if Raman and/or ATR-FTIR spectroscopy can detect these differences and indicate tumour origin. To contrast this, metastatic melanoma was selected since it provides a marked contrast in both appearances and immunohistochemical staining 
patterns to the adenocarcinomas (see Figure 1). The initial hypothesis was that the two adenocarcinoma groups would show similar spectral patterns and therefore would be difficult to differentiate as compared to the metastatic melanoma group, which

120 would demonstrate a marked difference. The novelty of this study lies in the

121 comparison of both Raman and FTIR-ATR within a pre-selected group of metastases,

122 with the analyses performed on spectral analysis without the need for complex 123 imaging.

\section{Methods}

125 Formalin-fixed paraffin embedded tissue from twenty-one brain metastasis

126 comprising colorectal adenocarcinoma metastasis $(n=7)$, lung adenocarcinomas metastasis $(n=7)$ and metastatic melanomas $(n=7)$ were obtained from the Brain Tumour North West (BTNW) research tissue bank (RTB - ethics NRES14/EE/1270).

129 Sections (10- $\mu$ m-thick) were placed onto glass slides covered with aluminium foil.

130 Foil-covered slides have been previously demonstrated to be as effective as more 131 expensive substrates significantly reducing the costs of this process (Cui et. al. 2016; 132 Paraskevaidi et al. 2018). These were de-waxed prior to spectral acquisition by 133 leaving overnight in fresh xylene. They were then washed in fresh xylene for $5 \mathrm{~min}$.

134 Following this, they were immersed in fresh ethanol at $100 \%$ twice and then $70 \%$ 135 ethanol once, for $5 \mathrm{~min}$ each, and then allowed to air dry prior to spectral acquisition. 136 H\&E-stained slides were viewed to delineate the tumour to be examined, to reduce contamination of spectra from background brain tissue. 
140 A Renishaw InVia Raman spectrometer was used to collect 25 spectra per section 141 using a $785 \mathrm{~nm}$ laser at $1200 \mathrm{~g} \mathrm{~mm}^{-1}$ grating with an acquisition time of 30 seconds

142 for each sample. This was over a spectral range of $400-1600 \mathrm{~cm}^{-1}$. A $50 \times$ objective 143 with numerical aperture of 0.85 was used to focus the laser beam. The spectral sites 144 were selected at random moving over the tissue. infrared spectrometer with Helios attenuated total reflection attachment containing a diamond crystal internal reflective element and a $45^{\circ}$ incidence angle of infrared beam. A new background spectrum was collected prior to each new sample, following cleaning of the crystal with distilled water. For each case 32 scans with $8 \mathrm{~cm}^{-1}$ spectral resolution were taken at 10 randomly selected points. The sampling aperture was $250 \mu \mathrm{m} \times 250 \mu \mathrm{m}$ and the mirror velocity was $2.2 \mathrm{~Hz}$.

\section{Computational analyses}

154 Computational analyses, including principal component analysis (PCA) with linear 155 discriminant analysis (LDA) and linear discriminant classifier (LDC) was then 156 performed within a MATLAB (Mathworks, Natick, USA) environment, using the 157 IRootlab toolkit as a user interface (Martin et al. 2010, Trevisan et. al. 2013, 158 Paraskevaidi et al. 2017). For classification spectra were pre-processed by cutting to 159 the region of interest $\left(\right.$ Raman $=500-1800 \mathrm{~cm}^{-1} ;$ ATR-FTIR $\left.=900-1800 \mathrm{~cm}^{-1}\right)$, 160 followed by polynomial baseline correction and vector normalisation. Spectra were 
then interrogated via PCA-LDA to generate scores plots and cluster vectors to determine points of variation between the spectra; PCA-LDC was then applied to calculate the classification accuracy as compared to the histopathological result. The top 6 spectral differences between the adenocarcinoma and melanoma groups were also determined.

\section{Results}

Analysis of the spectra has shown similar results for both Raman and ATR-FTIR spectroscopy. They demonstrate similar spectral appearances for both adenocarcinoma groups, with significant differences seen to the spectra of the melanoma. This can be seen primarily within both the pre-processed spectra [see Figure 2]. The lines for both adenocarcinoma groups show little variance, with the melanoma line clearly being separated at several points.

PCA-LDA was carried out to determine the principal components and thus the factors that account for most variance between the three groups in order to classify them. It was demonstrated that the groups show a degree of overlap (see Figure 3), which is greatest between the two adenocarcinoma groups. The points within the clusters show little difference within the adenocarcinoma groups, thought the melanoma group is clearly separated, with little overlap of the confidence bubbles. From this, cluster vectors were used to visualise the differences between the three groups. It can be seen (Figure 4) that the two adenocarcinoma groups are similar with small areas of variance (Figure $4 \mathrm{D} / \mathrm{d}$ ) as the lines are almost superimposed upon each other. However, the melanoma groups show a marked difference, with much greater separation of the two lines. This is particularly demonstrated within panel (D/d) where 
melanoma is taken as the baseline. This shows how similar adenocarcinomas are despite their different primary locations.

A PCA-LDC, giving the classification accuracy for each group as compared to the final histological diagnosis, was then performed (Figure 5). This was run for three separate groups and then two (combining the two adenocarcinoma groups) groups to show the difficulty in separating the adenocarcinomas. When using three groups for Raman, the classification accuracy is $69 \%$ for colorectal adenocarcinoma, $69 \%$ for lung adenocarcinoma and $72 \%$ for melanoma. Using ATR-FTIR spectroscopy this is $60 \%$ for colorectal adenocarcinoma, $59 \%$ for lung adenocarcinoma and $47 \%$ for melanoma. If the two adenocarcinoma groups are combined, classification accuracy markedly increases. With Raman spectroscopy this improves to $85 \%$ for adenocarcinoma and $75.4 \%$ for melanoma, and with ATR-FTIR spectroscopy $96 \%$ for adenocarcinoma and $72 \%$ for melanoma. This is, however, still below that found with traditional histopathology.

Following this, a one-way Anova was performed for the three groups to assess if the differences seen between the spectra were significant. A student's $t$-test was performed on the merged 2 groups to assess significance due to the small numbers involved (Figure 6). This was performed on the PCA-LDA results using all spectra for each case. For the three Raman spectroscopy groups this was $P=0.0016$ at $95 \%$ confidence interval and for ATR-FTIR spectroscopy this was not significant $(P=0.08)$ [see Supplementary information (SI) Table S1]. For two groups, this was again significant at $<0.0001$ for Raman and ATR-FTIR, with a 95\% confidence interval (see SI Table S2). 

Anova (see SI Table S1). This highlights the statistically significant differences found between adenocarcinoma and melanoma. There is no statistical difference between the two adenocarcinoma groups on either Raman or ATR-FTIR spectroscopy.

211 To conclude, the significant differences were calculated (see Figure 7) and tentative distinguishing wavenumbers assigned to those differences (Table 1). This was done to examine the points at which the tumours vary and to see which areas accounted for the variation. Within both Raman and ATR-FTIR spectroscopy the main variances were found within $\mathrm{CH}_{2}$ bond deformation and methylene twisting regions. Changes within these regions have previously been reported within carcinogenic samples (Movasaghi, Rehman and Rehman 2007, 2008) of varying types. Therefore, perhaps these regions are tied to carcinogenesis and not the particular tumour type with variations seen depending on the tumour.

\section{Discussion}

Both spectroscopic methods have been shown to be able to classify the different tumours by type (i.e., adenocarcinoma vs. melanoma), providing similar results. However, accuracy is greatly diminished if it is used to classify the primary origin of the tumour type, specifically determining if the adenocarcinoma arose within the lung or colon. Minor differences are seen between the spectra of these two tumours (see Figure 2); however, these differences are not statistically significant. This would, 227 therefore, limit any clinical use, as it would not be able to provide as much 228 information as traditional histopathology with H\&E and IHC. It may be that such new tools may aid the clinician in determining tumour type intra-operatively, i.e. that the tumour is a metastasis and not a primary brain tumour, but formal histopathology with 
231 IHC would still be required for primary tissue origin identification. This, however, is 232 also of interest given the marked spectral similarities between adenocarcinomas of 233 different primary origins (Figures 2 and 4). Within this study, confounding factors, 234 such as the number or location of the brain metastasis, nor patient factors have been 235 used to contribute to the accuracy of the results. As this was a comparison to conventional histopathology, these factors would not impact upon microscopy or immunohistochemistry, therefore it was felt not appropriate to be added into the 238 diagnostic algorithm.

239 When evaluating the potential value of spectroscopy as a possible intraoperative tool 240 its ability to determine cancer versus no cancer and suggest a tumour type would be 241 required. To provide further information to that provided by intraoperative neuropathology, spectroscopy would need to differentiate the primary tumour origin 243 for a metastasis. However, as can be seen, both Raman and ATR-FTIR spectroscopy 244 are able to detect differences between the two tumour types, but not specify the 245 primary tissue origin accurately enough for treatment decisions. As the technique 246 develops, it may replace frozen section, often performed intraoperatively to determine 247 if a tumour is primary, i.e., has arisen within the brain, or is a metastasis to guide the 248 surgeon in relation to the extent of the resection he may perform, as has been 249 suggested previously (Ji et. al. 2013, Ji et. al. 2015, Hollon et. al. 2016). At which 250 point, acknowledgement of a metastasis (from a primary tumour) would be the level required with histopathology completing the primary tumour origin determination as currently occurs. This would provide a potentially useful area for the technology to exploit as frozen section work can be challenging and potentially an area for error to

254 be removed by use of spectroscopy. However, comparative work to normal brain 
tissue and primary tumours would be required to ensure the technique is able to differentiate all potential results.

\section{Conclusion}

259

260 This study has highlighted both forms of spectroscopy are able to differentiate

261 different tumour types such as melanoma versus adenocarcinoma. However, it is not

262 able to differentiate tumour types to determine primary tissue origin of a metastasis in 263 its current form.

264 As the technique develops, it may eventually be able to provide additional 265 information to support the initial histopathological diagnosis, which may in the future 266 provide treatment related or prognostic information once the spectra are fully 267 understood in the years to come.

268 Conflicts of Interest The authors declare no conflicts of interest.

\section{Acknowledgements}

270 The authors would like to acknowledge the support from Rosemere Cancer 271 Foundation and the Brain Tumour North West RTB and the Sidney Driscoll 272 Neuroscience Foundation for their support.

273 Part of this work has been previously published as an abstract at the 2017 274 American Society of Clinical Oncology Meeting. J Clin Oncol 35, 2017 (suppl: 275 abstr e13551). 


\section{References}

Barr, H., C. Kendall, J. Hutchings, F. Bazant-Hegemark, N. Shepherd and N. Stone. 2011. Rapid endoscopic identification and destruction of degenerating Barrett's mucosal neoplasia. Surgeon, 9:119-123.

Bekaert, L., E. Emery, G. Levallet, and E. Lechapt-Zalcman. 2017. Histopathologic diagnosis of brain metastases: current trends in management and future considerations. Brain Tumour Pathology, 34:8-19.

Bergner, N., F. M. Romeike, R. Reichart, R. Kalff, C. Krafft, and J. Popp. 2013. Tumor margin identification and prediction of the primary tumour from brain metastases using FTIR imaging and support vector machines. Analyst, 138:39833990.

Cui, L., H. J. Butler, P. L. Martin-Hirsch, and F. L. Martin. 2016. Aluminium foil as a potential substrate for ATR-FTIR, transflection FTIR or Raman spectrochemical analysis of biological samples. Analytical Methods, 8:481-487.

Davis, F. G., T. A. Dolecek, B. J. McCarthy, and J. L. Villano. 2012. Toward determining the lifetime occurrence of metastatic brain tumours estimated from 2007 United States cancer incidence data. Neuro-Oncology, 14:1171-1177.

Gajjar, K., L .D. Heppenstall, W. Pang, K. M. Ashton, J. Trevisan, I. I. Patel, V. Llabjani, H. F. Stringfellow, P. L. Martin-Hirsch, T. Dawson, and F. L. Martin. 2012. Diagnostic segregation of human brain tumours using Fourier-transform infrared and/or Raman spectroscopy coupled with discriminant analysis. Analytical Methods, 5:89-102.

Hollon, T., S. Lewis, C. W. Freudiger, X. S. Xie, and D. A. Orringer. 2016. Improving the accuracy of brain tumor surgery via Raman-based technology. Neurosurgical Focus, 40:3-19. 
Huang, Q., and X. Ouyang. 2013. Predictive biochemical-markers for the development of brain metastases from lung cancer: clinical evidence and future directions. Cancer Epidemiology, 37:703-707.

Ji, M., D. A. Orringer, C. W. Freudiger, S. Ramkissoon, X. Liu, D. Lau, A. J.

Golby, I. Norton, M. Hayashi, N. Y. R. Agar, G. S. Young, C. Spino, S. Santagata,

S. Camelo-Piragua, K. L. Ligon, O. Sagher, and X. S. Xie. 2013. Rapid, label-free detection of brain tumours with stimulated Raman scattering microscopy. Science Translational Medicine, 5:201-224.

Ji, M., S. Lewis, S. Camelo-Piragua, S. H. Ramkissoon, M. Snuderl, S. Venneti, A. Fisher-Hubbard, M. Garrard, D. Fu, A. C. Wang, J. A. Heth, C. O. Maher, N. Sanai, T. D. Johnson, C. W. Freudiger, O. Sagher, X. S. Xie, and D. A. Orringer. 2015. Detection of human brain tumor infiltration with quantitative stimulated Raman scattering microscopy. Science Translational Medicine, 7:309-337.

Kalia, M. 2015 Biomarkers for personalized oncology: recent advances and future challenges. Metabolism Clinical and Experimental, 64:S16-21.

318 Kendall, C., J. Day, J. Hutchings, B. Smith, N. Shepherd, H. Barr, and N. Stone. 2010. Evaluation of Raman probe for oesophageal cancer diagnostics. Analyst, classification of IR spectroscopic images. Biochimica et Biophysica Acta, 1758:883-891.

Lee, D. K., J. H. Park, J. H. Kim, S. J. Lee, M. K. Jo, M. C. Gil, K. H. Song, and J. 
Lui, H., J. Zhao, D. McLean, and H. Zeng. 2012. Real-time Raman Spectroscopy for in vivo skin cancer diagnosis. Cancer Resources, 72:2491-2527.

Lyng, F. M., E. O’Faolain, J. Conroy, A, Meade, P. Knief, B. Duffy, M. B. Hunter, J. M. Byrne, P. Kelehan, and H. J. Byrne. 2007. Vibrational spectroscopy for cervical cancer pathology, form biochemical analysis to diagnostic tool, Experimental and Molecular Pathology, 82:121-129.

Martin, F. L., J. G. Kelly, V. Llabjani, P. L. Martin-Hirsch, I. I. Patel, J. Trevisan, N. J. Fullwood, and M. J. Walsh. 2010. Distinguishing cell types or populations based on the computational analysis of their infrared spectra. Nature Protocols, 5: 1748-1760.

Mitchell, A. L., K. B. Gajjar, G. Theophilou, F. L. Martin, and P. L. Martin-Hirsch. 2014. Vibrational spectroscopy of biofluids for disease screening or diagnosis: translation from the laboratory to a clinical setting. Journal of Biophotonics, 7:153165.

Movasaghi, Z., S. Rehman, and I. Rehman. 2007. Raman Spectroscopy of Biological Tissues. Applied Spectroscopy Reviews, 42:493-541.

Movasaghi, Z., S. Rehman, and I. Rehman. 2008. Fourier Transform Infrared (FTIR) Spectroscopy of Biological Tissues. Applied Spectroscopy Reviews, 43:134-179.

Owens, G. L., K. Gajjar, J. Trevisan, S. W. Fogarty, S. E. Taylor, B. Da GamaRose, P. L. Martin-Hirsch, and F. L. Martin. 2014. Vibrational biospectroscopy coupled with multivariate analysis extracts potentially diagnostic features in blood plasma/serum of ovarian cancer patients. Journal of Biophotonics, 7:200-209.

Paraskevaidi, M., C. L. M. Morais, K. M. G. Lima, J. S. Snowden, J. A. Saxon, A. M. T. Richardson, M. Jones, D. M. A. Mann, D. Allsop, P. L. Martin-Hirsch, and 
F. L. Martin. Differential diagnosis of Alzheimer's disease using spectrochemical analysis of blood. Proceedings of the National Academy of Sciences USA, 114: E7929-E7938.

Paraskevaidi, M., C. L. M. Morais, O. Raglan, K. M. G. Lima, E. Paraskevaidis, P. L. Martin-Hirsch, M. Kyrgiou, and F. L. Martin. 2018. Journal of Biophotonics, doi: 10.1002/jbio.201700372.

Renfrow, J. J., and G. J. Lesser. 2013. Molecular subtyping of brain metastases and implications for therapy. Current Treatment Options in Oncology, 14:514-527.

Sanghvi, S. M., J. W. Lischalk, L. Cai, S. Collins, M. Nair, B. Collins, and K. Unger. 2017. Clinical outcomes of gastrointestinal brain metastases treated with radiotherapy. Radiation Oncology, 12:43-51.

Theophilou, G., M. Paraskevaidi, K. M. Lima, M. Kyrgiou, P. L. Martin-Hirsch, and F. L. Martin. 2015. Extracting biomarkers of commitment to cancer development: potential role of vibrational spectroscopy in systems biology. Expert Review of Molecular Diagnostics, 15:693-713.

Theophilou, G., K. M. Lima, P. L. Martin-Hirsch, H. F. Stringfellow, and F. L. Martin. 2016. ATR-FTIR spectroscopy coupled with chemometric analysis discriminates normal, borderline and malignant ovarian tissue: classifying subtypes of human cancer. Analyst, 141:585-594.

Trevisan, J., P. P. Angelov, A. D. Scott, P. L. Carmichael, and F. L. Martin. 2013. IRootLab: a free and open-source MATLAB toolbox for vibrational biospectroscopy data anlaysis. Bioinformatics, 29:1095-1097. 
Table 1 The tentative assignments of significant points of difference for Raman and

378 attenuated total reflection-Fourier transform infrared (ATR-FTIR) spectroscopy,

379 using adenocarcinoma vs. melanoma (Movasaghi, Rehman and Rehman 2007, 2008).

\begin{tabular}{|c|c|c|}
\hline Method & Wavenumber $\left(\mathrm{cm}^{-1}\right)$ & Tentative assignment \\
\hline \multirow{7}{*}{ Raman } & & $\mathrm{CH}_{3} / \mathrm{CH}_{2}$ twisting or bending mode of lipid/collagen \\
\hline & 1310 & $\begin{array}{l}\mathrm{CH}_{3} / \mathrm{CH}_{2} \text { twisting, wagging \&/or bending mode of } \\
\text { collagens \& lipids }\end{array}$ \\
\hline & 1297 & $\mathrm{CH}_{2}$ deformation/Palmitic acid, acyl chains, fatty acids \\
\hline & 1296 & $\mathrm{CH}_{2}$ deformation \\
\hline & 1295 & Methylene twisting $/ \mathrm{CH}_{2}$ deformation \\
\hline & 1294 & Methylene twisting \\
\hline & 1293 & Cytosine/ Methylene twisting \\
\hline \multirow[t]{6}{*}{$\begin{array}{l}\text { ATR- } \\
\text { FTIR }\end{array}$} & 1720 & $\mathrm{C}=\mathrm{O}$ \\
\hline & 1578 & Ring C-C stretch of phenyl \\
\hline & 1481 & Amide II \\
\hline & 1477 & $\begin{array}{l}\mathrm{CH}_{2} \text { bending of methylene chains in lipids } \\
\text { /Polyethylene methylene of deformation modes }\end{array}$ \\
\hline & 1474 & $\begin{array}{l}\mathrm{CH}_{2} \text { bending of methylene chains in lipids } \\
\text { /Polyethylene methylene of deformation modes }\end{array}$ \\
\hline & 1470 & $\mathrm{CH}_{2}$ bending of methylene chains in lipids \\
\hline
\end{tabular}



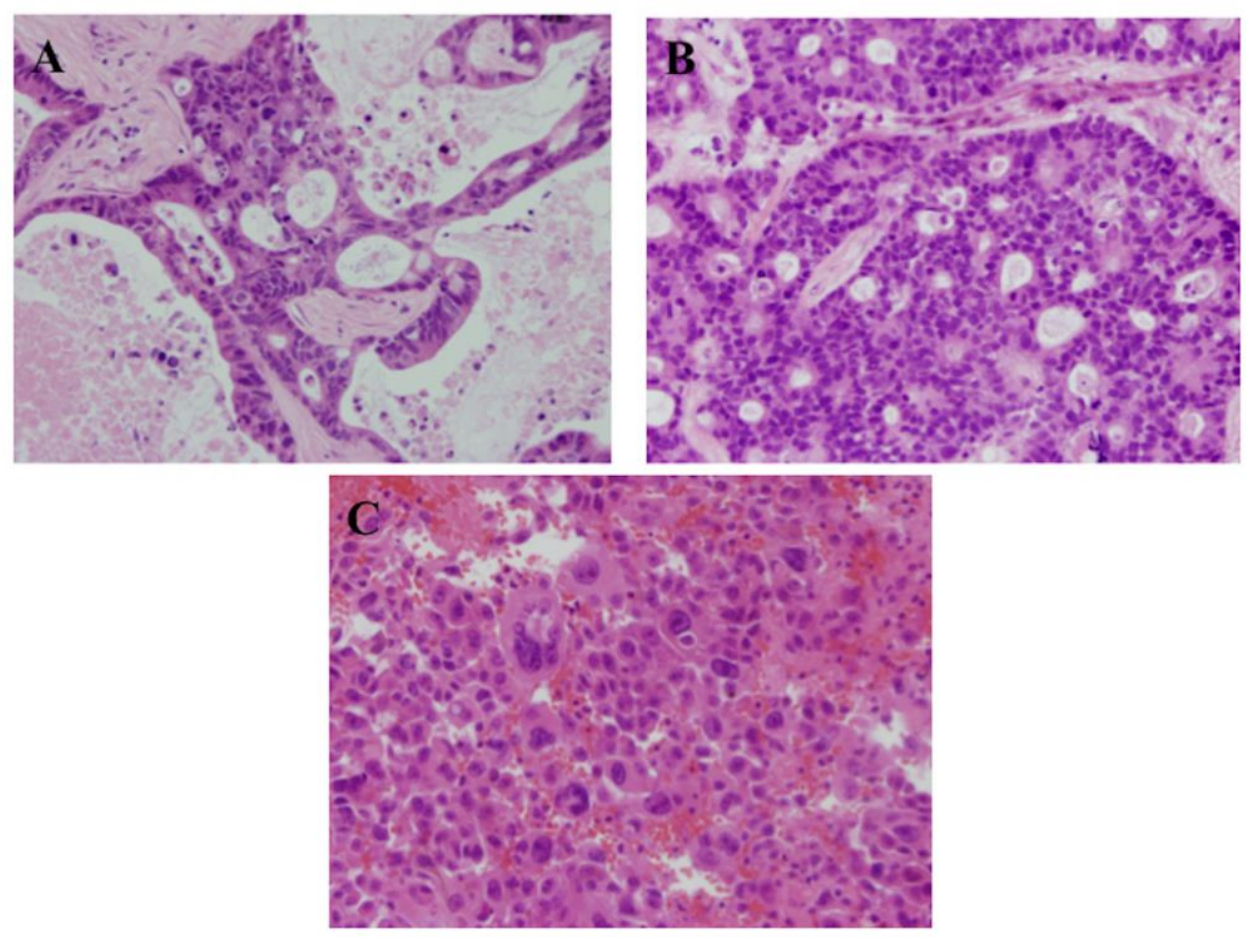

381 Figure 1 Representative photomicrographs of the microscopic appearance of brain 382 metastasis from different primary tumour sites. (A) is a metastasis from a colorectal 383 adenocarcinoma (H\&E $\times 200$ objective); (B) is a metastasis from a lung 384 adenocarcinoma (H\&E $\times 200$ objective); and, $(\mathrm{C})$ is a metastasis from a malignant 385 melanoma $(\mathrm{H} \& \mathrm{E} \times 200$ objective $)$. 

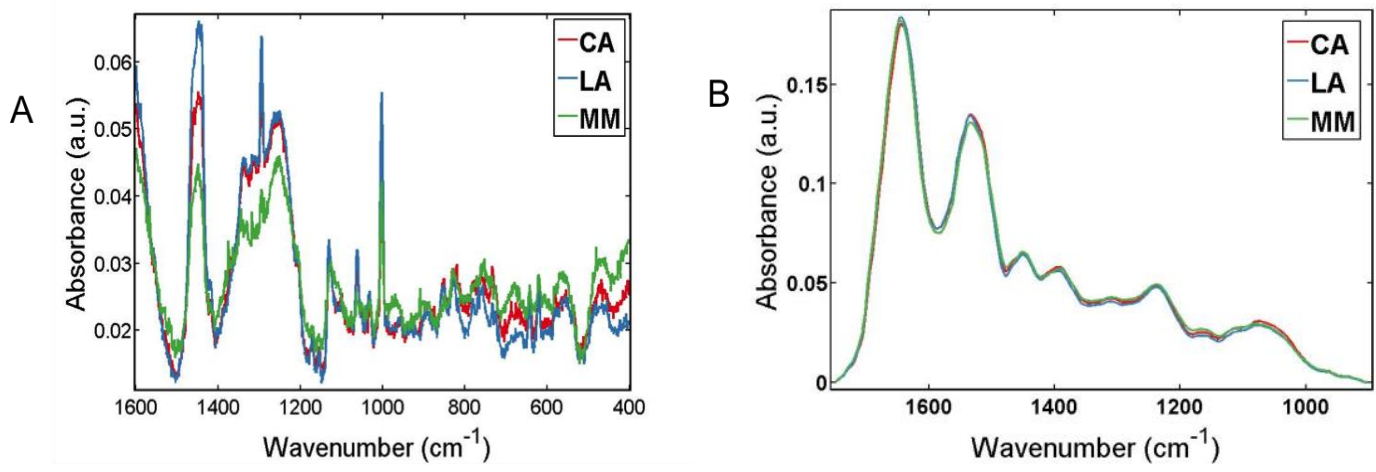

388 Figure 2 A graph demonstrating the mean pre-processed spectra from each tumour

389 group using: (A) Raman spectroscopy (cut to the region of interest, polynomial 390 baseline correction and vector normalisation); and, (B) ATR-FTIR spectroscopy (cut 391 to the region of interest, rubberband baseline correction and vector normalisation).

392 (KEY: CA=COLORECTAL ADENOCARCINOMA, LA=LUNG ADENOCARCINOMA,

393 MM=MELANOMA).

394

395 
A

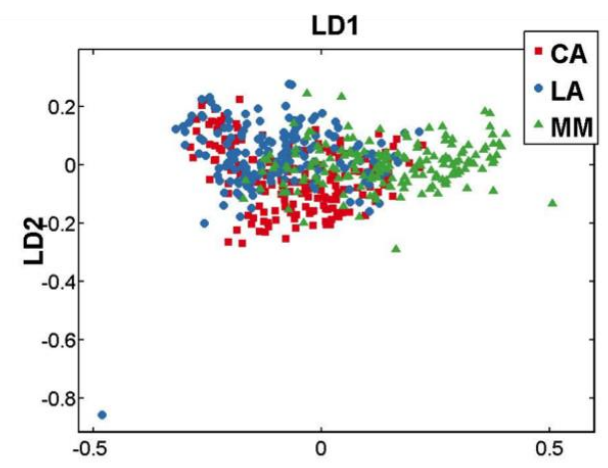

396

C

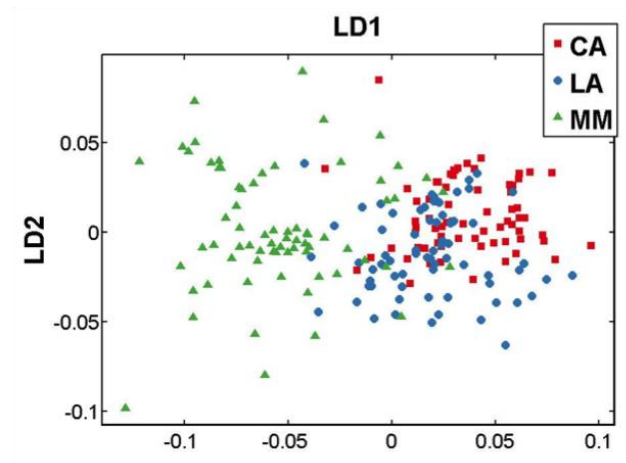

B

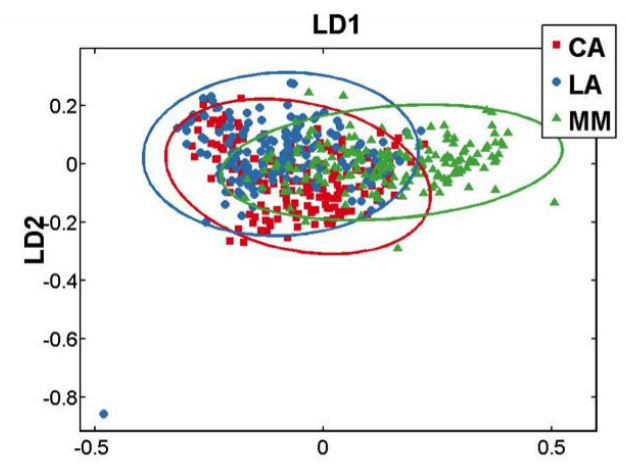

D

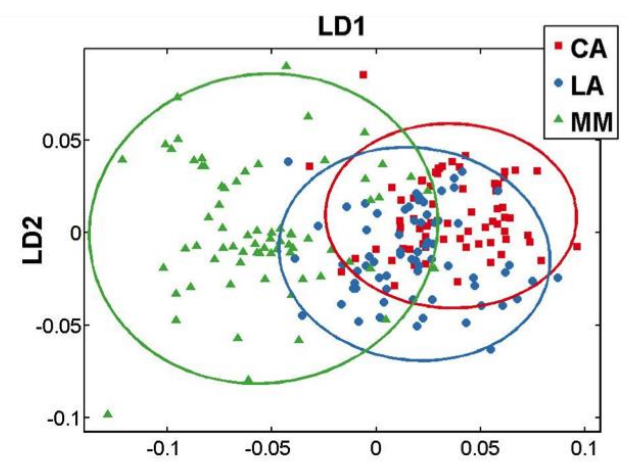

Figure 3 A graph demonstrating the PCA-LDA results for Raman and ATR-FTIR

399 spectroscopy. The left side demonstrates the Raman spectroscopy results firstly

400 without (A) and secondly with (B) 95\% confidence intervals. This is then mirrored on

401 the right for ATR-FTIR spectroscopy, without (C) and with (D) 95\% confidence

402 intervals. (KEY: CA - COLORECTAL ADENOCARCINOMA, LA - LUNG

403

ADENOCARCINOMA, MM - MALIGNANT MELANOMA) 
A

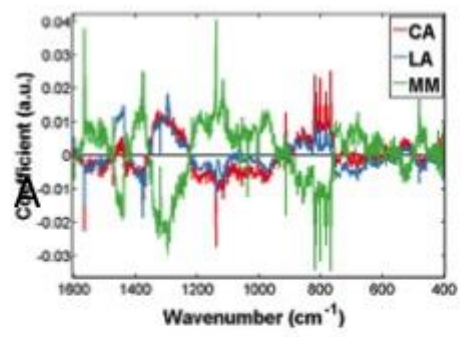

B

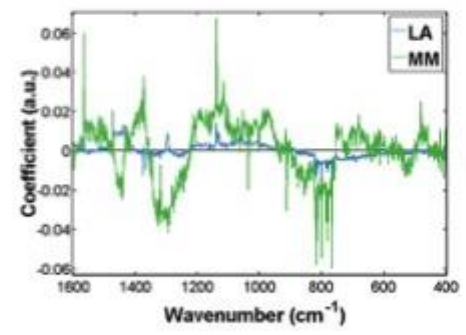

C

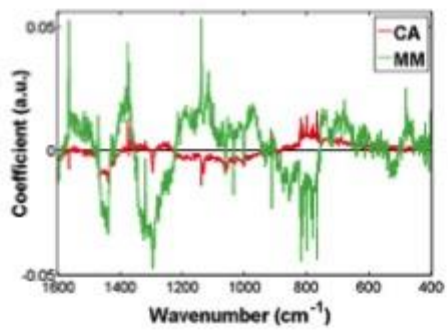

D

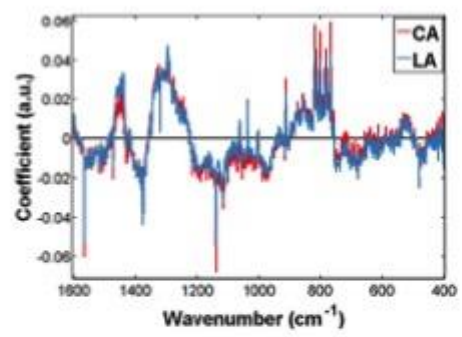

E

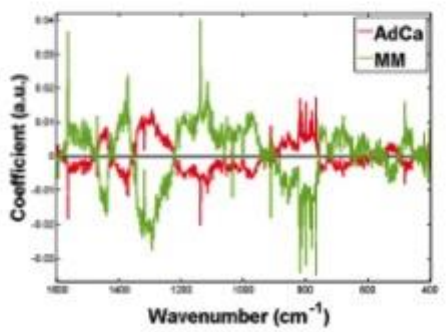

a

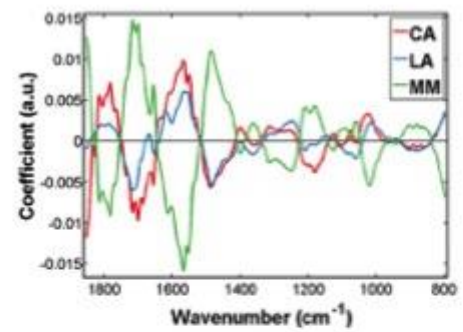

b

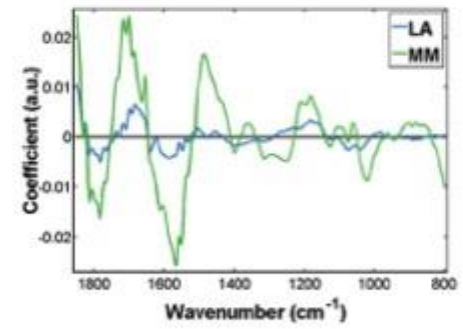

C

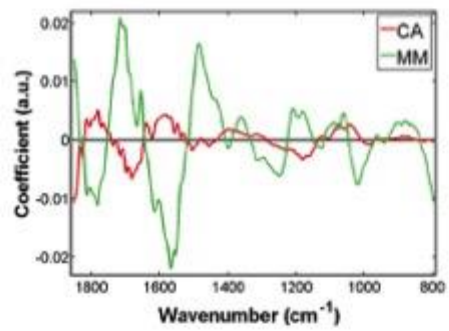

d

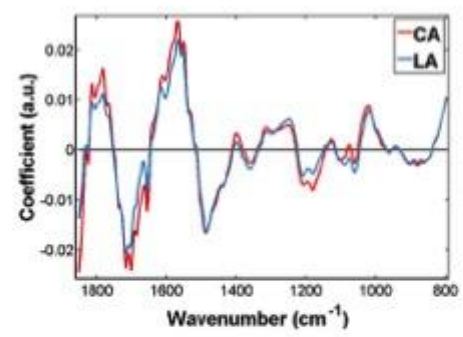

e

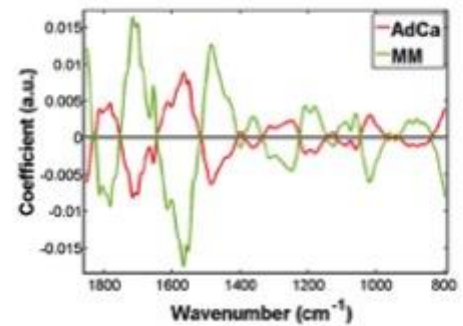

Figure 4 These graphs show the cluster vectors for Raman and ATR-FTIR

409 spectroscopy. The upper case displays the Raman spectroscopy results, starting 410 with (A/a) all the groups, $(\mathrm{B} / \mathrm{b}) \mathrm{CA}$ is taken as the baseline, $(\mathrm{C} / \mathrm{c}) \mathrm{LA}$ taken as 411 the baseline, (D/d) MM taken as baseline and (E/e) compares adenocarcinoma 

vs. MM. This is mirrored on the right, with lower case letters for ATR-FTIR

413 spectroscopy. (KEY: CA - COLORECTAL ADENOCARCINOMA, LA - LUNG 414 ADENOCARCINOMA, MM - MALIGNANT MELANOMA, AdCA 415 ADENOCARCINOMA).

416 
A

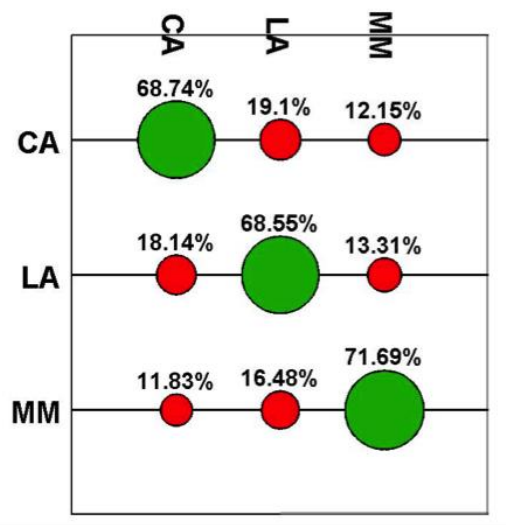

B

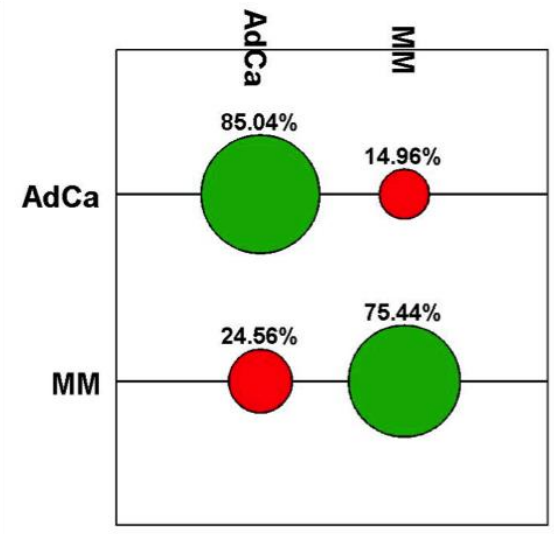

C

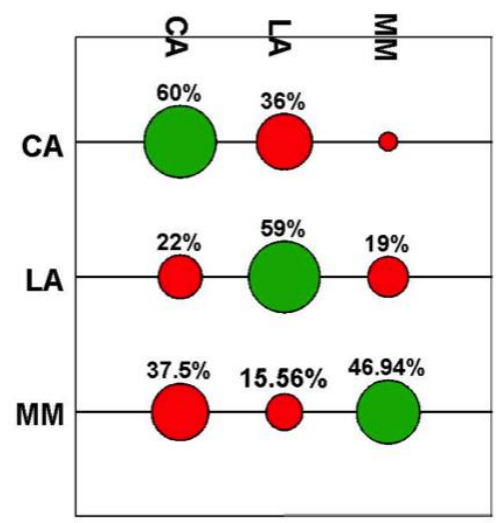

D

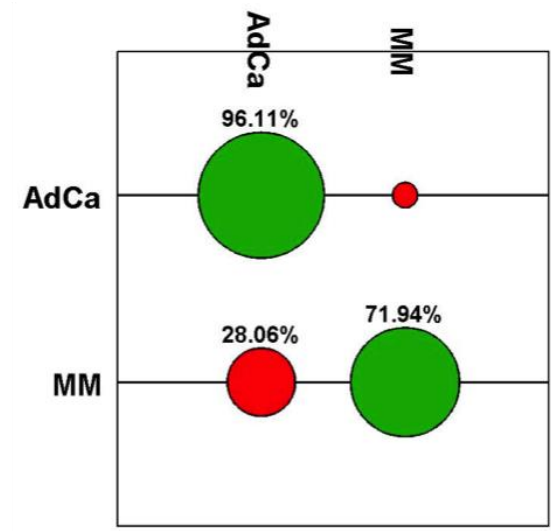

419 correct group (green) or another group (red). The Raman results are shown on the left

420 with (A) displaying each of the three cancer groups separately, and (B) compares

421 adenocarcinoma to MM. On the right are the ATR-FTIR spectroscopy results; (C)

422 displays each of the three cancer groups separately and (D) again compares

423 adenocarcinoma to MM. (KEY: CA - COLORECTAL ADENOCARCINOMA, LA -

424 Lung ADENOCARCINOMA, MM - MALIGNANT MELANOMA, AdCA -

425 ADENOCARCINOMA). 
A

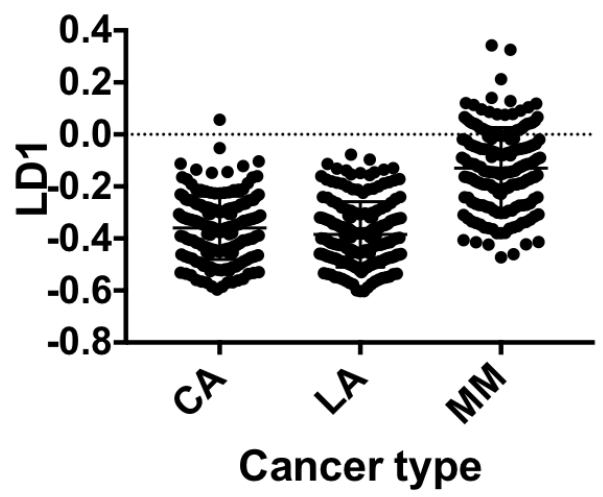

B

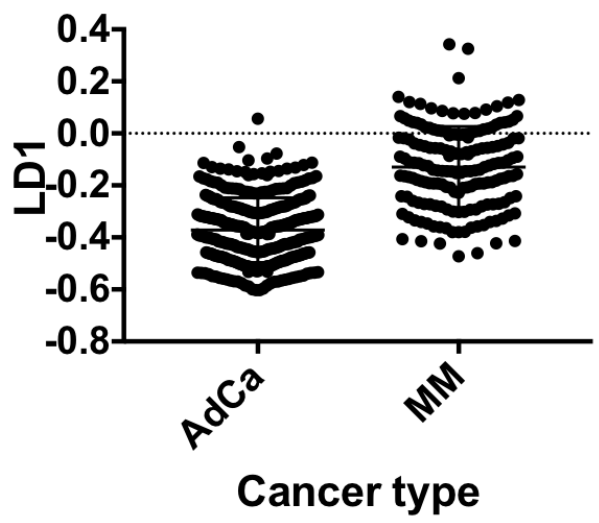

C

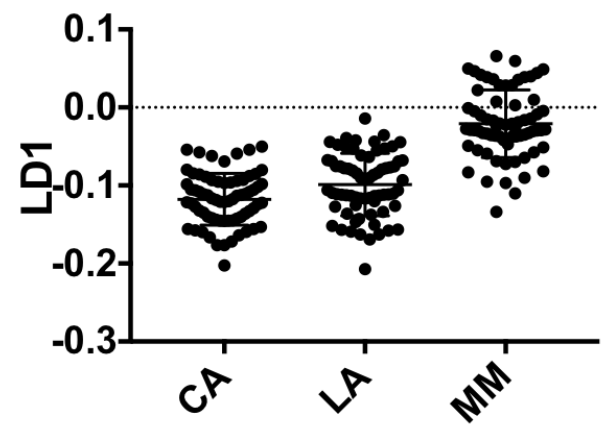

Cancer type

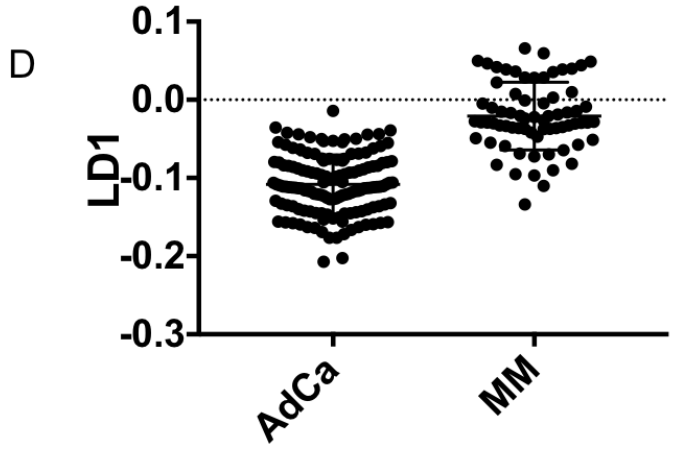

Cancer type

427 Figure 6 These graphs represent the results of both a one-way Anova and student's $t$ -

428 test scores plot for Raman and ATR-FTIR spectroscopy. (A) shows the one-way

429 Anova for Raman with all three tumour groups, (B) the student's $t$-test for Raman

430 spectroscopy with adenocarcinoma and MM. This is mirrored for ATR-FTIR

431 spectroscopy with (C) showing the one-way Anova for ATR-FTIR spectroscopy with

432 all three tumour groups and (D) the student's $t$-test for ATR-FTIR spectroscopy with

433 adenocarcinoma and MM. (KEY: CA - COLORECTAL ADENOCARCINOMA, LA -

434 LUNG ADENOCARCINOMA, MM - MALIGNANT MELANOMA). 
A

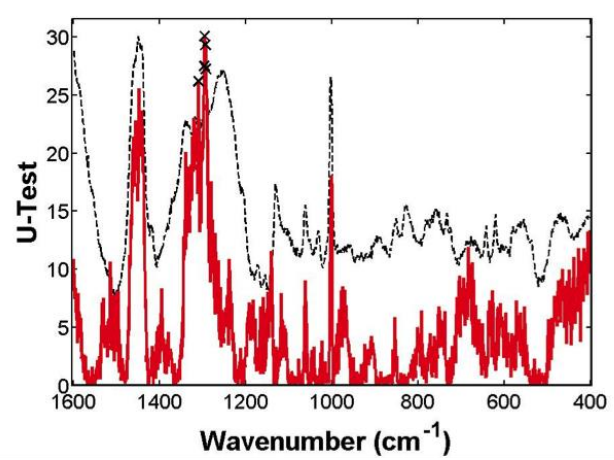

B

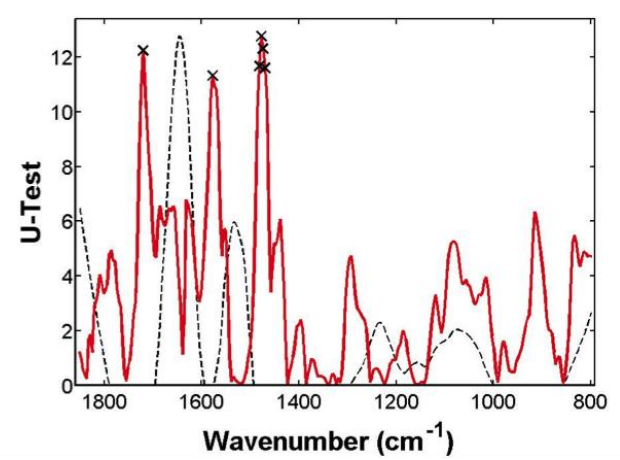

436 Figure 7 The significant wavenumber differences between the adenocarcinoma

437 groups and melanoma. (A): Raman spectroscopy, (B): ATR-FTIR spectroscopy 